\title{
Correction to: Abundance of the common vampire bat and feeding prevalence on cattle along a gradient of landscape disturbance in southeastern Mexico
}

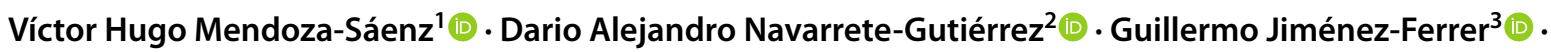 \\ Cristian Kraker-Castañeda ${ }^{1,4}$ (i) $\cdot$ Romeo Alberto Saldaña-Vázquez ${ }^{5}$
}

Published online: 10 August 2021

(c) Mammal Research Institute, Polish Academy of Sciences, Bialowieza, Poland 2021

\section{Correction to: Mammal Research \\ https://doi.org/10.1007/s13364-021-00572-9}

In the original published version of this article, the labels in the image of Figures 2, 5 and 6 were not presented during publication. The correct images has been uploaded.

The original article has been corrected.

Publisher's note Springer Nature remains neutral with regard to jurisdictional claims in published maps and institutional affiliations.

The original article can be found online at https://doi.org/10.1007/ s13364-021-00572-9

Víctor Hugo Mendoza-Sáenz

vhmendoza@ecosur.edu.mx

Dario Alejandro Navarrete-Gutiérrez

dnavarre@ecosur.mx

Guillermo Jiménez-Ferrer

gjimenez@ecosur.mx

Cristian Kraker-Castañeda

ckraker@ecosur.edu.mx

Romeo Alberto Saldaña-Vázquez

romeoalberto.saldana@iberopuebla.mx

1 Departamento de Conservación de La Biodiversidad, El Colegio de La Frontera Sur (ECOSUR), Carretera Panamericana y Periférico Sur S/N, Barrio María Auxiliadora, 29290 San Cristóbal de Las Casas, Chiapas, Mexico

2 Grupo Académico: Ecología, Paisaje y Sustentabilidad. Departamento: Observación y Estudio de la Tierra, la Atmósfera y el Océano, El Colegio de La Frontera Sur (ECOSUR), Carretera Panamericana y Periférico Sur S/N, Barrio María Auxiliadora, 29290 San Cristóbal de Las Casas, Chiapas, Mexico

3 Departamento de Agricultura, Sociedad y Ambiente, El Colegio de La Frontera Sur (ECOSUR), Carretera Panamericana y Periférico Sur S/N, Barrio María Auxiliadora, 29290 San Cristóbal de Las Casas, Chiapas, Mexico

4 Centro de Estudios Conservacionistas (CECON), Unidad para el Conocimiento, Uso y Valoración de la Biodiversidad, Universidad de San Carlos de Guatemala, Avenida Reforma 0-63, Zona 10, 01010 Guatemala City, Guatemala

5 Instituto de Investigaciones en Medio Ambiente Xabier Gorostiaga, S.J., Universidad Iberoamericana Puebla, Colonia Reserva Territorial Atlixcáyotl, Boulevard del Niño Poblano No. 2901, 72820 San Andrés Cholula, Puebla, Mexico 